\title{
Obliterative bronchiolitis after kidney transplantation: A
} case report

\author{
Anthony O’Riordan, Sophie Gregg, Deirdre Doyle, Aisling Farrell, Barry J Plant, Michael Clarkson and Desmond M Murphy \\ Consultant Respiratory Physician and Clinical Lecturer, Cork University Hospital and University College Cork, National Clinical Lead in Respiratory Medicine, \\ Ireland
}

\begin{abstract}
A 61-year-old gentleman developed obliterative bronchiolitis 12 years after deceased donor kidney transplantation. This was diagnosed based on progressive dyspnoea, severe airflow obstruction and typical radiographic features. Despite management with long-term oxygen therapy and pulmonary rehabilitation, the patient died roughly 3 years after diagnosis. Obliterative bronchiolitis is potentially an under-recognised late complication in patients after kidney transplantation.
\end{abstract}

\begin{abstract}
Abbreviations: BOS: bronchiolitis obliterans syndrome; ESKD: end-stage kidney disease; BO: bronchiolitis obliterans; OB: obliterative bronchiolitis; ISHLT: International Society for Heart and Lung Transplantation; RA: rheumatoid arthritis
\end{abstract}

\section{Case Report}

We present the case of a 61-year-old gentleman who developed bronchiolitis obliterans (BO)/obliterative bronchiolitis (OB) 12 years after deceased donor kidney transplant. His end-stage kidney disease (ESKD) was due to glomerulonephritis and he had been on haemodialysis for 6 years before transplantation. He had no episodes of acute rejection post-transplant and had stable graft function.

Eleven years after transplant he developed worsening, progressive dyspnoea. He was dyspnoeic on minimal exertion with associated functional decline. He had no previous personal or family history of pulmonary disease. He was a retired dairy and beef farmer. $\mathrm{He}$ was a non-smoker. His medications at the time of presentation were Azathioprine 50mg BD, Prednisolone 5mg OD, Ramipril 7.5mg OD and Omeprazole 20mg OD. On examination, his $\mathrm{SpO} 2$ was $85 \%$ on room air, he was tachypnoeic and on auscultation of his lungs he had globally decreased intensity of breath sounds.

Initial investigations showed an arterial pO2 of $6.5 \mathrm{kPa}$ on room air. Serum samples showed a normal cell count and stable chronic kidney disease with an eGFR of $33 \mathrm{ml} / \mathrm{min} / 1.73 \mathrm{~m}^{2}$. Autoantibodies and serum precipitants to farmer's lung antigens were negative. Chest radiograph was unremarkable. CT thorax with contrast showed extensive bronchial wall thickening and mosaic attenuation characteristic of OB (Figures 1 and 2). There was no consolidation or effusion. Pulmonary function tests were performed and demonstrated a severe obstructive defect with an FEV1 of $0.48 \mathrm{~L}$ (16\% predicted).

Transthoracic echocardiogram showed a mildly dilated right ventricle and mild systolic dysfunction. He had a right heart catheterisation which showed pulmonary hypertension with a mean pulmonary artery pressure of $33 \mathrm{mmHg}$ and a mean pulmonary capillary wedge pressure of $12 \mathrm{mmHg}$. He also had a coronary angiogram which showed normal coronary arteries.

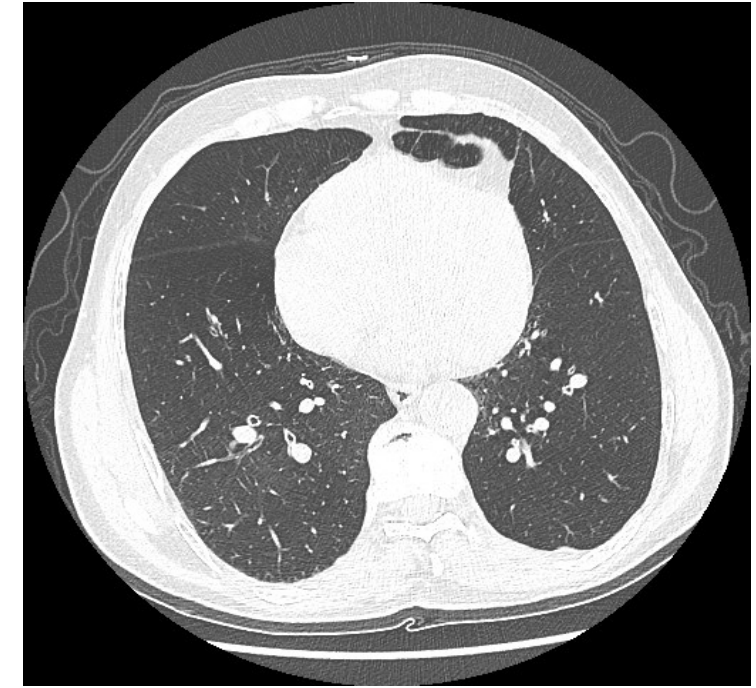

Figure 1. CT thorax shows airway thickening and mosaic attenuation

Bronchoalveolar lavage was performed which showed evidence of acute inflammation with no malignant cells, no specific organisms and was negative for pneumocystis. Viral and bacterial cultures were negative. Transbronchial biopsy did not show diagnostic features of obliterative bronchiolitis.

Given his clinical and radiographic features of $O B$, he was commenced on long-term oxygen therapy. He was started on PO

${ }^{\star}$ Correspondence to: Desmond Murphy, MB, PhD, FCCP, FRCPI, FRCPEdin, Consultant Respiratory Physician and Clinical Lecturer, Cork University Hospital and University College Cork, National Clinical Lead in Respiratory Medicine, Ireland, E-mail: Desmond.Murphy@hse.ie

Key words: obliterative bronchiolitis, bronchiolitis obliterans syndrome, kidney transplant

Received: December 17, 2020; Accepted: December 24, 2020; Published: December 28, 2020 


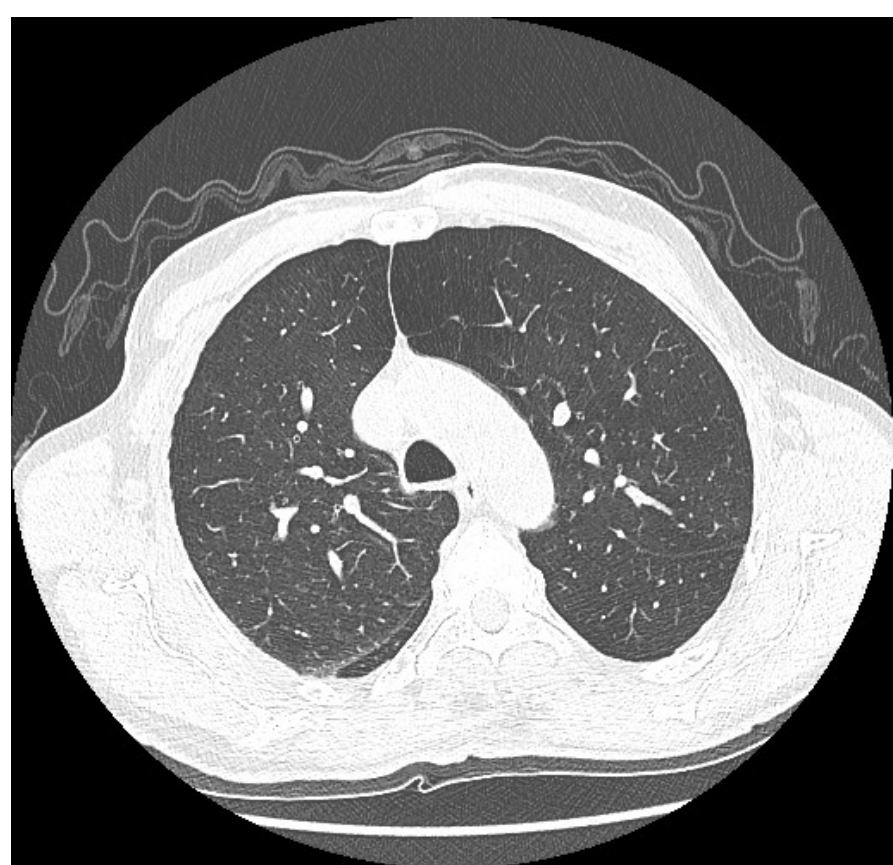

Figure 2. CT thorax shows airway thickening and mosaic attenuation

Azithromycin and nebulised bronchodilators. He made very limited progress with pulmonary rehabilitation.

Over the next 3 years, his hypoxia and dyspnoea progressed despite therapy. He succumbed to respiratory failure at the age of 66 years.

\section{Discussion}

To the best of our knowledge, there are no reported cases of OB after kidney transplant. In patients post lung transplant, chronic allograft rejection manifests histologically as OB. In 2002, the International Society for Heart and Lung Transplantation (ISHLT) proposed a clinical description of $\mathrm{OB}$, termed bronchiolitis obliterans syndrome (BOS) which is defined by pulmonary function changes rather than histology [1]. BOS is defined as a progressive airflow obstruction, with a decrease in FEV1 of $20 \%$ or greater from the best postoperative value, in the absence of anastomotic stricture, infection, bronchitis or other complications [2]. BOS carries a poor prognosis and results in progressive respiratory failure.

OB has generally been reported to occur in three patient populations. Firstly, BOS is among the commonest causes of late graft failure in lung transplantation. In adult deceased donor lung transplants, BOS affects $80 \%$ of recipients at 10 years from transplant [3]. OB is also associated with connective tissue disease; particularly rheumatoid arthritis (RA). This is usually seen in patients with severe RA late in the disease but has been described as the sole manifestation of RA [4]. Lastly, OB can also be seen post allogeneic hematopoietic cell transplant (HCT). It affects approximately $2-3 \%$ of all allogeneic HCT recipients and is thought to be a manifestation of chronic graft versus host disease (GVHD) [5].
While the pathophysiology of $\mathrm{OB}$ is poorly defined, it is increasingly recognized that a common-pathway response to bronchial epithelial injury may occur with epithelial activation, inflammation and an excessive epithelial-mesenchymal fibroblastic repair response resulting in OB [1]. It appears to progress through a sequence of lymphohistiocytic-mediated cytotoxicity directed at the respiratory epithelium [2]. While OB has not been reported as a complication of kidney transplantation, fibrocytes produced in the bone marrow targeting the allograft may result in a similar process when directed at the respiratory epithelium. The histological description of $\mathrm{OB}$ shows the obliteration or respiratory bronchioles with increased deposition of scar collagen [6].

Risk factors currently identified for BOS after lung transplant include donor and recipient age, graft ischemia, reperfusion injury; but there are also shared risk factors amongst patients post allogeneic hematopoietic cell transplant or with connective tissue disease. These include aspiration, use of immunosuppressant medications, chronic inflammation and recurrent microbial infections. A pulmonary response to immunosuppressive therapy or chronic cytomegalovirus (CMV) infection could explain the mechanism by which OB is seen across these population groups.

While previously described as irreversible, recent work suggests low-dose macrolides could reverse the airflow obstruction seen in BOS [7]. We believe this is an important case to highlight a possible late complication of solid organ transplant. As survival rates following solid organ transplantation continue to improve, $\mathrm{OB}$ may become a more commonly reported in this population [8].

\section{References}

1. Estenne M, Maurer JR, Boehler A, Egan JJ, Frost A, et al. (2002) Bronchiolitis obliterans syndrome 2001: an update of the diagnostic criteria. J Heart Lung Transplant 21: 297-310. [Crossref]

2. Robertson AGN, Griffin SM, Murphy DM, Pearson JP, Forrest IA, et al. (2009) Targeting Allograft Injury and Inflammation in the Management of Post-Lung Transplant Bronchiolitis Obliterans Syndrome. American Journal of Transplantation 9: $1272-1278$.

3. https://ishltregistries.org/registries/slides.asp

4. Schwarz MI, Lynch DA, Tuder R (1994) Bronchiolitis obliterans: the lone manifestation of rheumatoid arthritis? European Respiratory Journal 7: 817-820.

5. Chien JW, Duncan S, Williams KM, Pavletic SZ (2010) Bronchiolitis obliterans syndrome after allogeneic hematopoietic stem cell transplantation - an increasingly recognized manifestation of chronic graft-versus-host disease. Biol of Blood Marrow Transplant 16: S106-S114. [Crossref]

6. Vanaudenaerde BM, Meyts I, Vos R, Geudens N, De Wever W, et al. (2008) A dichotomy in bronchiolitis obliterans syndrome after lung transplantation revealed by azithromycin therapy. European Respiratory Journal 32: 832-842.

7. Yates B, Murphy DM, Forrest IA, Ward C, Rutherford RM, et al. (2005) Azithromycin reverses airflow obstruction in established bronchiolitis obliterans syndrome. American journal of respiratory and critical care medicine 172: 772-775.

8. Ruttens D, Verleden SE, Demeyer H, Van Raemdonck DE, Yserbyt J, et al. (2018) Montelukast for bronchiolitis obliterans syndrome after lung transplantation: a randomized controlled trial. PloS one 13: e0193564. [Crossref]

Copyright: (2020 O’Riordan A. This is an open-access article distributed under the terms of the Creative Commons Attribution License, which permits unrestricted use, distribution, and reproduction in any medium, provided the original author and source are credited. 